\title{
Oral administration of an immunodominant $T$-cell epitope downregulates Th1/Th2 cytokines and prevents experimental myasthenia gravis
}

\author{
Fulvio Baggi, ${ }^{1}$ Francesca Andreetta, ${ }^{1}$ Elisabetta Caspani, ${ }^{1}$ Monica Milani, ${ }^{1}$ \\ Renato Longhi, ${ }^{2}$ Renato Mantegazza, ${ }^{1}$ Ferdinando Cornelio, ${ }^{1}$ and Carlo Antozzi ${ }^{1}$ \\ ${ }^{1}$ Department of Neuromuscular Diseases, National Neurological Institute "C. Besta," 20133 Milan, Italy \\ ${ }^{2}$ Institute of Biocatalysis and Molecular Recognition, Consiglio Nazionale delle Ricerche, 20131 Milan, Italy \\ Address correspondence to: Carlo Antozzi, Department of Neuromuscular Diseases, \\ National Neurological Institute "C. Besta," Via Celoria 11, 20133 Milan, Italy. \\ Phone: 39-022394255; Fax: 39-0270633874; E-mail: car.antozzi@xquasar.it.
}

Received for publication April 19, 1999, and accepted in revised form September 22, 1999.

The mucosal administration of the native antigen or peptide fragments corresponding to immunodominant regions is effective in preventing or treating several $\mathrm{T}$ cell-dependent models of autoimmune disease. No data are yet available on oral tolerance with immunodominant T-cell peptides in experimental autoimmune myasthenia gravis (EAMG), an animal model of B cell-dependent disease. We report that oral administration of the T-cell epitope $\alpha 146-162$ of the Torpedo californica acetylcholine receptor (TAChR) $\alpha$-subunit suppressed T-cell responses to AChR and ameliorated the disease in $\mathrm{C} 57 \mathrm{Bl} / 6$ (B6) mice. Protection from EAMG was associated with reduced serum Ab's to mouse AChR and reduced AChR loss in muscle. The effect of T $\alpha 146-162$ feeding was specific; treatment with a control peptide did not affect EAMG manifestations. The protective effect induced by peptide To146-162 was mediated by reduced production of IFN- $\gamma$, IL-2, and IL-10 by TAChR-reactive cells, suggesting T-cell anergy. TGF- $\beta$-secreting Th3 cells did not seem to be involved in tolerance induction. We therefore demonstrate that feeding a single immunodominant epitope can prevent an $\mathrm{Ab}$ mediated experimental model of autoimmune disease.

J. Clin. Invest. 104:1287-1295 (1999).

\section{Introduction}

Ideal treatment of autoimmune diseases should inhibit the immune response against target antigens specifically and permanently without interfering with the general function of the immune system. A way to induce antigenspecific tolerance at the T-cell level is by administering the relevant antigen through either the oral or nasal routes $(1,2)$. This approach has been investigated extensively in T-cell mediated animal models, particularly experimental autoimmune encephalomyelitis (EAE), that were successfully tolerized by the mucosal delivery of the native antigen or its peptide fragments (1,3-4).

Experimental autoimmune myasthenia gravis (EAMG) is an animal model of myasthenia gravis (MG), a B cell-mediated, T cell-dependent autoimmune disease of the neuromuscular junction (5) suitable for use in investigating new therapeutic strategies. Data available on the structure and epitope mapping of the acetylcholine receptor (AChR) allow the design of new approaches potentially able to modulate the autoimmune response in EAMG, a prototypical Abmediated disease in which $\mathrm{AChR}$ recognition by $\mathrm{CD} 4^{+}$ T lymphocytes has been well characterized.

Specific suppression of EAMG was first induced in rabbits with a denatured preparation of the AChR (6). More recently, EAMG was prevented in rats by the oral and nasal administration of purified Torpedo californica
AChR (TAChR) (7-11). However, when the effect of TAChR feeding was investigated in ongoing EAMG in Lewis rats, not only was the degree of protection partial but Ab's to AChR were increased compared with control animals (10). Such a boosting effect on autoantibody levels induced by feeding native TAChR after immunization suggested the need for less immunogenic molecules such as AChR $\alpha$-subunit recombinant fragments (12) or synthetic peptides.

The AChR $\alpha$-subunit plays a major role in the pathogenesis of EAMG and likely in MG (5). CD4 ${ }^{+} \mathrm{T}$ cells from $\mathrm{C} 57 \mathrm{Bl} / 6$ (B6) mice immunized with TAChR recognize different regions of the TAChR $\alpha$-subunit, corresponding to the sequences T $\alpha 111-126$, T $\alpha 146-162$,

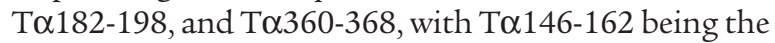
immuo-dominant T-cell epitope $(13,14)$. The immunodominance of this sequence was further confirmed in the EAMG-resistant bm12 strain of mice: $\mathrm{T}$ cells from TAChR-primed bm12 mice showed very low proliferative responses to peptide T $\alpha 146-162$ compared with B6 mice (15). The identification of immunodominant $\mathrm{T}$ cell epitopes on the AChR molecule suggests their potential use as substitutes for the native antigen in tolerization studies. EAMG has been prevented in B6 mice by the subcutaneous administration of peptide T $\alpha 146-162(16,17)$ and by the nasal delivery of a pool of TAChR $\alpha$-subunit peptides containing this epitope (18). 


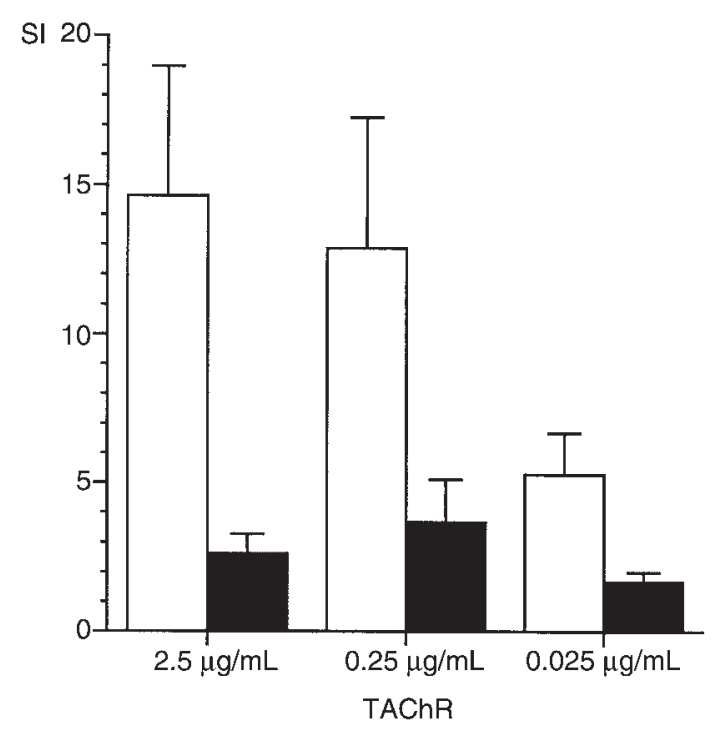

Figure 1

Oral administration of purified TAChR suppresses antigen-specific T-cell responses in B6 mice. Mice were given $1 \mathrm{mg} \times 4$ doses of TAChR orally every other day and were immunized with TAChR 2 days after the last feeding. Mice were sacrificed 10 days after immunization for T-cell proliferation studies. Data are represented as $\mathrm{SI} \pm$ SEM. Open columns: PBS-treated mice $(n=6)$; filled columns: TAChR-treated mice $(n=5)$. The effect of TAChR feeding on T-cell proliferation was statistically significant $(P<0.05)$ at 2.5 and 0.025 $\mu \mathrm{g} / \mathrm{mL}$ of TAChR. The average cpm of cultured LN cells in the absence of antigen were $277 \pm 59$ for PBS-treated and $404 \pm 125$ for TAChR-treated mice.

To our knowledge, no data are yet available on the oral administration of this immunodominant epitope in murine EAMG. We report that the oral administration of peptide T $\alpha 146-162$ in B6 mice suppressed T-cell responses to itself, to a subdominant epitope, and to the whole TAChR and prevented the clinical manifestations of the disease. Peptide treatment reduced significantly pathogenic Ab's to self-AChR and muscle AChR loss. We also report on the effect of peptide treatment on Th1-, Th2-, and Th3-associated cytokines in our model.

\section{Methods}

Antigens. TAChR was purified from electroplax tissue by affinity chromatography with Naja-naja siamensis toxin (19). The specific activity was $3.7-5.5 \mathrm{nmol}$ of $\left[{ }^{125} \mathrm{I}\right] \alpha$-bungarotoxin binding sites $(\alpha$-BTX-binding sites) per milligram of protein, and its purity was verified by SDS-PAGE. TAChR preparations were extensively dialyzed against Tris-EDTA buffer, $0.1 \%$ Triton $\mathrm{X}-100$, and stored at $-80^{\circ} \mathrm{C}$. TAChR used for T-cell proliferation was further dialyzed against Tris-EDTA buffer, $0.025 \%$ Triton, and was filter sterilized. Peptides corresponding to sequences To111-126 (DYTGKIMWTPPAIFKS), T $\alpha 146-162$ (LGIWTYDGTKVSISPES), and T $\alpha 182-198$ (RGWKHWVYYTCCPDTPY) of the TAChR $\alpha$-subunit, as well as a control peptide (sequence 751-765 of human complement factor $\mathrm{H}$, KEFDHNSNIRYRCRG, Swiss-Prot P08603) were synthesized using f-moc chemistry on an Applied Biosys- tems 431A automated peptide synthesizer (Applied Biosystems Perkin-Elmer Europe B.V., Monza, Italy).

Immunization and treatment protocols. Female B 6 mice, 6 to 7 weeks of age, were purchased from Charles River Italia (Calco, Italy). All experiments were approved by the Scientific Board of the National Neurological Institute.

Two groups of experiments were performed to investigate (a) the effect of oral feeding with TAChR or peptide T $\alpha 146-162$ on primary antigen-specific responses from lymph node $\mathrm{T}$ cells (T-cell tolerance) and (b) the induction of tolerance to TAChR by feeding B6 mice with To146-162 peptide before TAChR immunization and 2 subsequent boosts necessary to induce EAMG (prevention of EAMG). Animals were deprived of food before each feeding, and TAChR or peptide were administered by gavage under ether anesthesia. Mice were fed with 1 $\mathrm{mg}$ TAChR or 2 different doses $(0.5 \mathrm{mg}$ or $1 \mathrm{mg})$ of To146-162 in a volume of $400 \mu \mathrm{L}$ of PBS 4 times every other day before immunization; control animals received PBS or the control peptide. No inhibitors of proteolytic enzymes were used. Two days after the last feeding, mice were immunized by injection in the hind footpads with $20 \mu \mathrm{g}$ of TAChR in CFA (day 0). EAMG was induced with 2 TAChR boosts at day 30 and 75 (20); each boost was preceded by peptide feeding as described above.

Lymphocyte proliferation assay. Primary antigen-specific responses were studied from draining lymph node (LN) T cells of peptide and PBS-fed mice 10 days after immunization. T-cell proliferation was also studied from the spleens 1 month after the second boost (day 105). LN and spleens were aseptically removed and processed into single-cell suspensions. T cells from each mouse were cultured in RPMI-1640 medium containing $100 \mu \mathrm{g} / \mathrm{mL}$ streptomycin, $100 \mu \mathrm{g} / \mathrm{mL}$ penicillin, $2 \mathrm{mM}$ L-glutamine, $1 \mathrm{mM}$ sodium pyruvate (GIBCO BRL-Life Technologies Italia, Milan, Italy), and 10\% FCS (Sigma-Aldrich, Milan, Italy). Concanavalin A (Sigma-Aldrich) was used at $4 \mu \mathrm{g} / \mathrm{mL}$ as positive control. T lymphocytes were challenged with $\operatorname{TAChR}(2.5,0.25$, and $0.025 \mu \mathrm{g} / \mathrm{mL})$ or peptide $(50,10$, and $1 \mu \mathrm{g} / \mathrm{mL}$ ). After 72 hours, $\left[{ }^{3} \mathrm{H}\right]$-thymidine (Amersham Italia, Milan, Italy) at $1 \mu \mathrm{Ci} /$ well was added to triplicate wells; cells were harvested after 16 hours. Uptake of $\left[{ }^{3} \mathrm{H}\right]$-thymidine was measured by beta counting. Results are expressed either as stimulation index (SI; ratio between the mean of cpm from antigen-stimulated culture and the mean of cpm from unstimulated culture) or as mean cpm.

Clinical evaluation of EAMG. EAMG evaluation was performed as follows: EAMG score: grade 0, normal strength; grade 1 , normal appearance at rest, fatigability induced by repetitive paw grips on the cage grid; grade 2 , signs of weakness at rest; grade 3 , moribund; and grade 4, dead. Muscle fatigability was expressed as the time taken for each mouse to release its hold from an inverted grid; the test was arbitrarily stopped when the holding time exceeded 12 minutes. Results are expressed as the mean of 3 different evaluations for each animal (adapted from ref. 18). The test was performed blindly. 

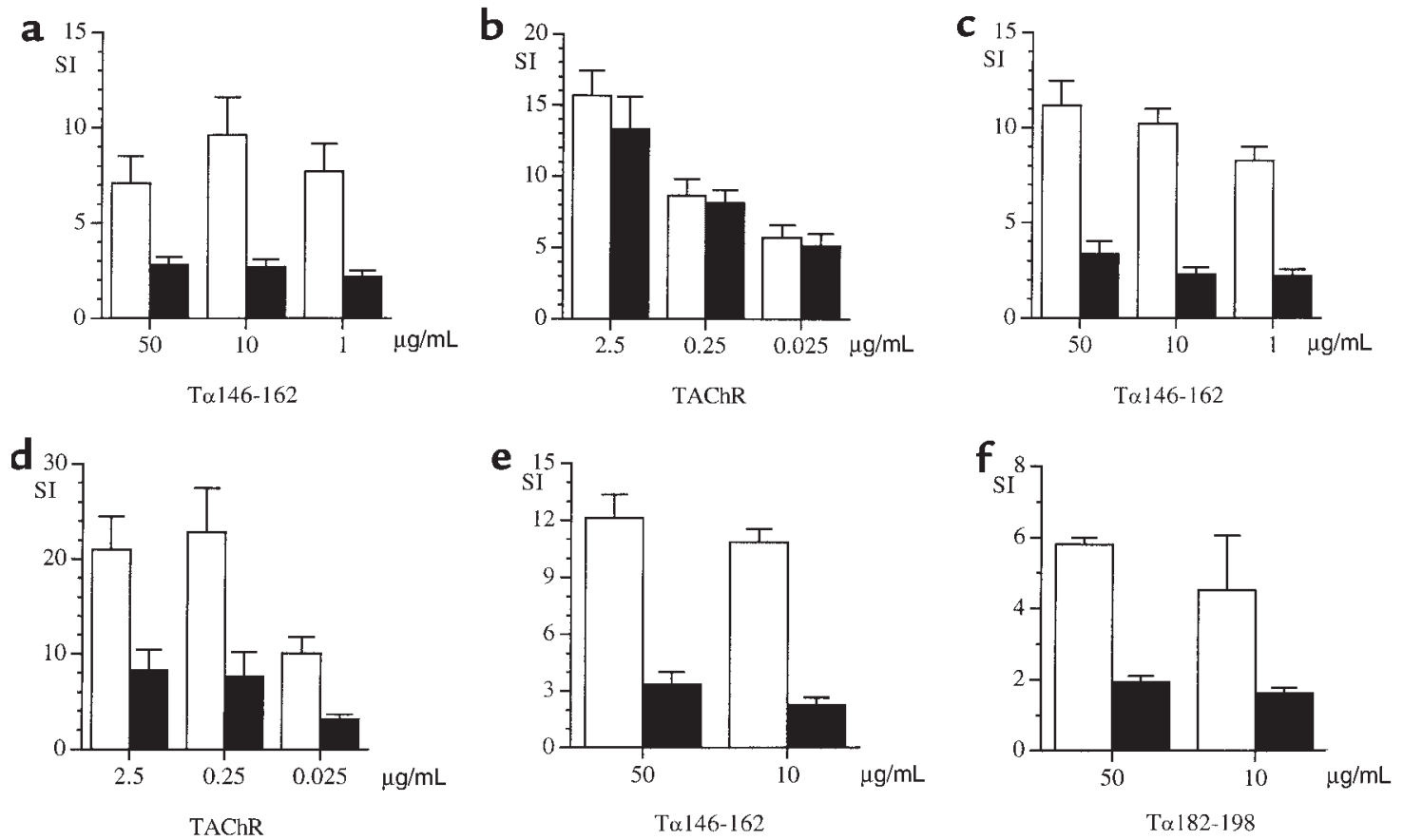

\section{Figure 2}

Oral administration of peptide T $\alpha 146-162$ suppresses T-cell responses to T $\alpha 146-162$, TAChR, and the subdominant epitope T $\alpha 182-198$. Open columns: PBS-treated mice; filled columns: T $\alpha 146-162$-treated mice. T-cell proliferation was assayed 10 days after immunization: 0.5 $\mathrm{mg} \times 4$ doses of peptide T $\alpha 146-162$ reduced T-cell proliferation to the peptide itself $(P<0.02)(\mathbf{a})$; no differences were noted when LN cells were challenged with TAChR (b). On the contrary, when $1 \mathrm{mg} \times 4$ doses of T $\alpha 146-162$ was given, T-cell proliferation was significantly reduced to both T $\alpha 146-162, P<0.001$ (c), or TAChR, $P<0.02$ (d). Moreover, T cells from peptide-fed $(1 \mathrm{mg} \times 4$ doses) or PBS-fed mice showed a significant reduction of proliferation to T $\alpha 146-162, P<0.05(\mathbf{e})$, and to the subdominant epitope T $\alpha 182-198, P<0.001$ (f). Data are represented as mean SI \pm SEM. The average cpm of cultured LN cells in the absence of antigen were $1121 \pm 220$ for PBS-treated and $1719 \pm 520$ for T $\alpha 146-162$-treated mice (a and b); $141 \pm 26$ for PBS-treated and $203 \pm 30$ for T $\alpha 146-162-$ treated mice (c and d); $160 \pm 35$ for PBS-treated and $300 \pm 45$ for $T \alpha 146-162$-treated mice (e and $\mathbf{f}$ ).

Anti-AChR Ab assay. Anti-TAChR and anti-mouse AChR Ab's were assayed by the conventional radioimmunoassay procedure (21). Briefly, serial dilutions (up to $5 \mu \mathrm{L}$ ) of experimental (peptide and vehicle-fed) and control mice sera were incubated overnight at $4^{\circ} \mathrm{C}$ with 0.5 pmol TAChR labeled with [ $\left.{ }^{125} \mathrm{I}\right] \alpha$-BTX. Rabbit antimouse IgG (DAKO A/B, Glostrup, Denmark) was added $(50 \mu \mathrm{L})$ for 4 hours at room temperature. The pellets were washed twice with cold PBS $1 \%$ Triton X100 and counted in a gamma counter. For mouseAChR Ab's assay, experimental sera were added to mouse AChR labeled with $\left[{ }^{125} \mathrm{I}\right] \alpha$-BTX and incubated overnight at $4^{\circ} \mathrm{C}$. Ab-AChR complexes were precipitated with rabbit anti-mouse IgG for 4 hours. Pellets were washed twice with $1 \mathrm{~mL}$ cold PBS buffer, $1 \%$ Triton X100. Serum samples incubated with mouse AChR preincubated in excess of cold $\alpha$-BTX (aspecific binding) were subtracted from test samples. Ab titers were expressed as picomoles of toxin-binding sites per milliliter of mouse serum.

Determination of anti-AChR Ab isotypes. IgG isotypes of anti-TAChR Ab's (total IgG, IgG1, IgG2, IgG2a, and $\operatorname{IgG} 2 b$ ) were determined on pooled sera collected from animals treated with peptide T $\alpha 146-162$ or PBS, as reported above for anti-AChR Ab assay using secondary Ab's against mouse IgG isotypes (Sigma-Aldrich).
AChR content in mouse muscle. Mouse AChR was extracted from the whole mouse carcass. Each carcass was weighed and homogenized for 1 minute at high speed in 4 volumes of Tris buffer. AChR was solubilized from membranes with Triton X-100 (10\%) in Tris buffer, for 4 hours at $4^{\circ} \mathrm{C}$, then centrifuged at 100,000 $g$ for 30 minutes $\left(4^{\circ} \mathrm{C}\right)$. Duplicate $0.1-\mathrm{mL}$ aliquots of mouse muscle AChR crude extract were incubated with an excess of [ $\left.{ }^{125} \mathrm{I}\right] \alpha$-BTX, transferred to a DE-81 DEAE disk and washed with Triton X-100 (0.5\%) Tris buffer. Radioactivity was determined by gamma counting. Aspecific binding (from parallel tubes preincubated with unlabeled $\alpha$-BTX) was subtracted from each sample. Results were expressed as picomoles of toxin-binding sites per gram of carcass.

Cytokine assay. Capture ELISAs for detection of mouse IL-2, IFN- $\gamma$, IL-4, IL-6, IL-10 (Genzyme Pharmaceuticals, Cambridge, Massachusetts, USA), and TGF $\beta$ (Promega Corp., Madison, Wisconsin, USA) were performed according to manufacturers' recommendations. LN or splenic T cells $\left(1.5 \times 10^{6} / \mathrm{mL}, 24-\right.$ well plates) were cultured in the presence of 2.5 $\mu \mathrm{g} / \mathrm{mL}$ TAChR in complete RPMI-1640 medium. FCS was not added for TGF- $\beta$ assay. Supernatants were harvested at appropriate times for each cytokine and stored at $-80^{\circ} \mathrm{C}$. 

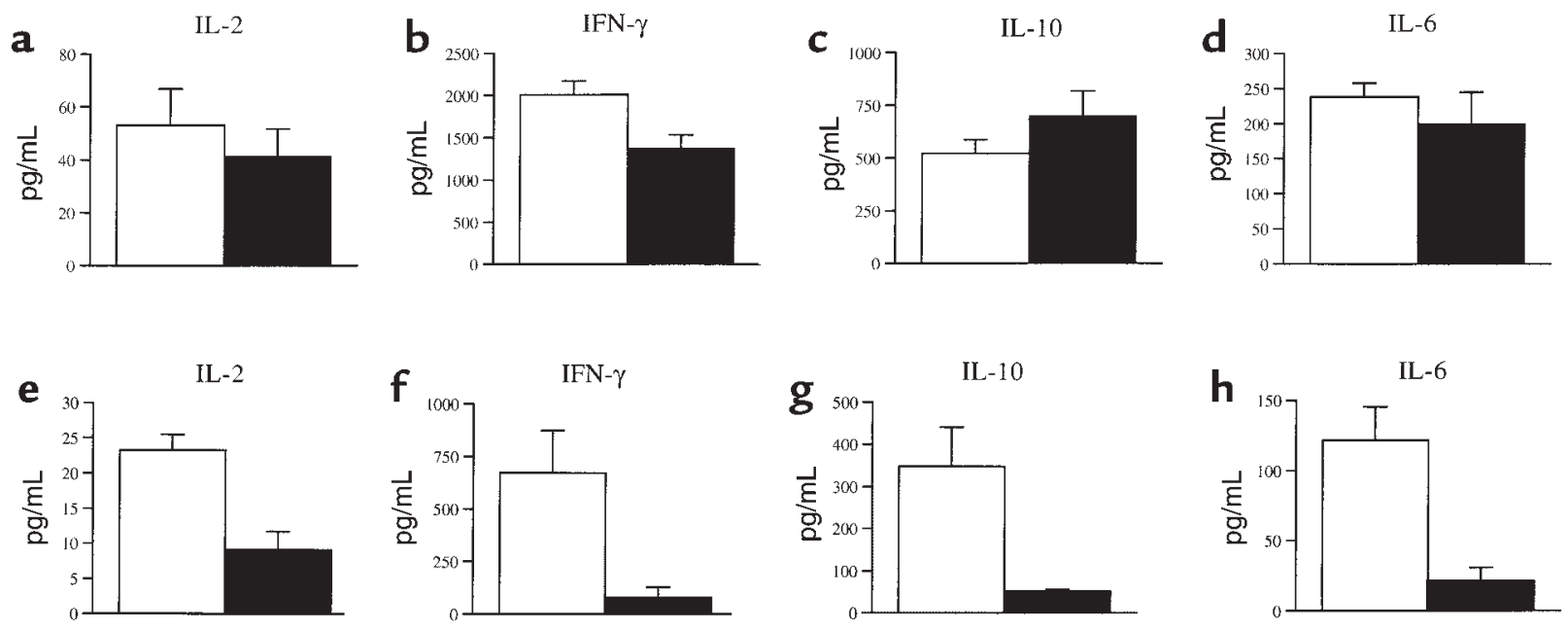

Figure 3

T-cell tolerance to AChR induced by peptide T $\alpha 146-162$ is associated with a dose-related reduction of Th1 and Th2 cytokines. Th1 (IL-2 and IFN- $\gamma$ ) and Th2 (IL-10 and IL-6) were assayed in cell culture supernatants from mice treated with peptide To146-162 and immunized with TAChR 2 days after the last feeding; animals were sacrificed 10 days after immunization, and LN cells were challenged in vitro with TAChR. (a-d) Animals were given $0.5 \mathrm{mg} \times 4$ doses of peptide To146-162; no significant differences were noted between peptide-treated (filled bars) and PBS-treated mice (open bars), except for a significant reduction of IFN- $\gamma(P<0.02)$. The increase of IL-10 levels was not statistically significant. $(\mathbf{e}-\mathbf{h})$ The experiment performed with $1 \mathrm{mg} \times 4$ doses of peptide gave a significant reduction of all the cytokines tested in LN cell supernatants $(P<0.02)$

RT-PCR for detection of TGF- $\beta$ mRNA in Peyer's patches. Total RNA was extracted from Peyer's patches (PP) by the RNAWIZ RNA isolation reagent (Ambion Inc., Austin, Texas, USA). Random hexamer cDNA was prepared from $5 \mu \mathrm{g}$ of total RNA using reverse transcriptase. The resulting cDNA mixture was used directly in a specific PCR amplification with the addition of specific primers. The sequences were as follows: TGF- $\beta$ forward, 5'-AGTGTGGAGCAAC ATGTGGA-3'; TGF- $\beta$ reverse, $5^{\prime}$ GTGAGCGCTGAATCGAAAG-3'; G6PDH forward, $5^{\prime}$-CGA CTCACAGTGGACGACAT -3'; G6PDH reverse 5'TCTCTTTGCCCAGGTAGT GGTC- $3^{\prime}$. Thirty-five cycles were performed $\left(94^{\circ} \mathrm{C}\right.$ denaturation for 1 minute, $55^{\circ} \mathrm{C}$ annealing for 1 minute, $72^{\circ} \mathrm{C}$ extension for 1 minute). Amplified products were analyzed by electrophoresis on $2 \%$ agarose gels stained with ethidium bromide (ET). The ET fluorescence of the two bands (TGF- $\beta$ and G6PDH as reference transcript) was quantified by the Kodak Digital Science 1D software (Eastman Kodak Co. Scientific Imaging Systems, New Haven, Connecticut, USA), and expressed as the ratio between the net intensity of TGF- $\beta$ and that of G6PDH band.

Statistical analysis. Results from treated and control groups were compared by 2 -tailed Student's $t$ test using Statview 4.5 (Abacus Concepts Inc., Berkeley, California, USA).

\section{Results}

Oral administration of peptide Ta146-162 suppresses T-cell responses to To146-162 and to TAChR in a dose-related fashion. When $1 \mathrm{mg}$ per dose of TAChR was administered 4 times every other day without trypsin inhibitor, T-cell proliferation was significantly suppressed (Figure 1), showing that T-cell tolerance to TAChR can be obtained by feeding the native antigen to $\mathrm{B} 6$ mice. However, the high amount of antigen needed for these studies suggested that synthetic fragments containing TAChR epitopes could be used in further investigation.

LN T cells from TAChR-immunized mice challenged

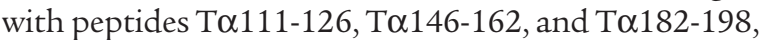
indicated that the epitope To146-162 (SI = 11.2 \pm 1.3 at $50 \mu \mathrm{g} / \mathrm{mL}$ ) was the immunodominant epitope, compared with $\mathrm{T} \alpha 182-198(\mathrm{SI}=5.0 \pm 0.7)$ and $\mathrm{T} \alpha 111-126$ $(\mathrm{SI}=3.2 \pm 1.9$ ), hence was the candidate of choice for oral tolerization studies.

Four doses of $0.5 \mathrm{mg}$ of peptide were administered every other day before immunization. Ten days later, animals were sacrificed and the reactivity of LN T cells was measured in vitro against T $\alpha 146-162$. Peptide feeding reduced significantly $\mathrm{T}$-cell proliferation (54-65\%, depending on the antigen concentration; Figure 2a). However, the proliferative response to TAChR was not modified compared with PBS-fed mice (Figure 2b). On the basis of this finding, mice were treated with $1 \mathrm{mg}$ T $\alpha 146-162$ per dose: a significant inhibition of T-cell responses was observed not only to peptide Ta146-162 (Figure 2c) but also to TAChR (Figure $2 \mathrm{~d}$ ). These results suggested that the effect of peptide Ta146-162 on TAChR-specific T-cell responses was probably dose related.

Oral administration of peptide To146-162 suppresses T-cell proliferation to the subdominant epitope To182-198. We tested whether the oral administration of peptide To146-162 was able to reduce $\mathrm{T}$-cell responses against the subdominant epitope To182-198. Treatment with peptide To146$162(1 \mathrm{mg} \times 4$ every other day) reduced significantly T-cell responses against $T \alpha 146-162$ itself and to the subdominant epitope To182-198 also (Figure 2, e and f). 
Therefore, T $\alpha 146-162$ treatment affected T-cell reactivity to a subdominant epitope as well as to the whole native antigen (Figure $2 \mathrm{~d}$ ), presumably involving a mechanism of tolerance spreading. These findings further supported the feasibility of oral tolerization studies on clinical EAMG with peptide T $\alpha 146-162$.

T-cell tolerance to AChR induced by peptide T $\alpha 146-162$ is associated with reduction of Th1 and Th2 cytokines. To investigate the mechanisms associated with T $\alpha 146-162$-induced Tcell tolerance, we evaluated Th1-type (IL-2 and IFN- $\gamma$ ) and Th2-type (IL-4, IL-6, and IL-10) cytokines in culture supernatants of LN T cells stimulated in vitro with TAChR. We could not find any significant modification of cytokine levels in mice treated with $0.5 \mathrm{mg}$ per dose of peptide T $\alpha 146-162$, except for IFN- $\gamma$, the reduction of which was statistically significant (Figure 3, a-d). Note that with this dose of peptide, T-cell proliferation was reduced only when $\mathrm{LN} T$ cells were challenged with the peptide itself, but not with TAChR (Figure 2, a and b). On the contrary, the suppression of TAChR-specific T-cell responses obtained by feeding $1 \mathrm{mg}$ per dose of peptide T $\alpha 146-162$ was associated with a significant reduction of IL-2, IFN- $\gamma$, IL-6, and IL-10 (Figure 3, e-h). IL-4 was undetectable in both peptide-fed and PBS-fed animals.

Oral treatment with peptide T $\alpha 146-162$ ameliorates clinical EAMG, and the effect is antigen specific. Because the higher dose of peptide To146-162 induced T-cell tol-
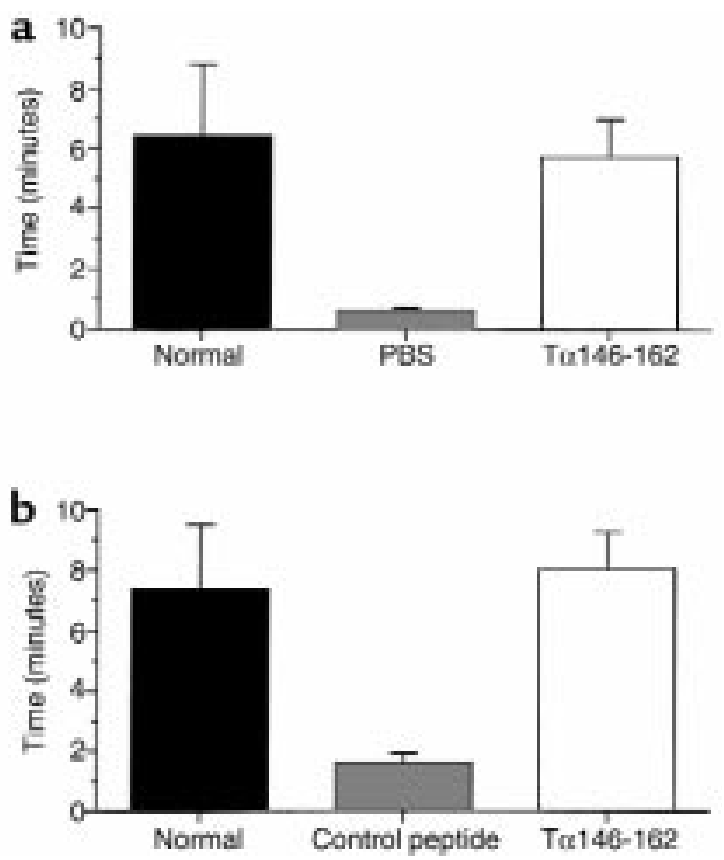

Figure 4

Oral treatment with peptide T $\alpha 146-162$ protects from clinical EAMG. Evaluation of mice fatigability in different treatment groups by the inverted hang test. The evaluation performed at the end of the experiments is reported. A group of normal mice was included for comparison in each experiment. The mean holding times ( \pm SEM) for PBS- and T $\alpha 146-162$-treated mice were $0.6 \pm 0.1$ minutes and $5.7 \pm$ 1.3 , respectively $(P=0.009)(\mathbf{a})$. The mean holding times for control peptide- and T $\alpha 146-162$-treated mice were $1.6 \pm 0.3$ minutes and $8.0 \pm 1.2$, respectively $(P<0.001)(\mathbf{b})$. erance to the AChR, we studied its effect on the clinical manifestations of the disease. EAMG was evaluated by a clinical score and by an inverted grid test as reported in Methods. No deaths due to EAMG occurred in the peptide-fed group $(n=12)$, whereas 3 mice died of EAMG in the PBS-fed group $(n=10)$. No overt clinical signs of EAMG were observed in peptide-treated animals whereas the mean clinical score $( \pm$ SEM) of PBS-treated mice was $1.9 \pm 0.53$. To confirm the specificity of the protective effect of our peptide, we repeated the experiment and compared the effect of T $\alpha 146-162$ with an unrelated control peptide. No deaths occurred in the T $\alpha 146-162$-treated group $(n=12)$, whereas 2 mice died of EAMG in the control peptide group $(n=12)$. The mean clinical score was $0.42 \pm 0.15$ in T $\alpha 146-162$-treated animals and $1.5 \pm 0.4$ in control peptide-fed mice $(P=0.014)$. Moreover, we evaluated the degree of fatigability in normal mice, PBS-fed animals, and peptide-treated animals, expressed as the holding time from an inverted grid. As shown in Figure 4a, peptide-treated mice were protected from EAMG fatigability compared with PBS-fed animals. The mean holding time of peptide-fed mice was comparable to that of normal mice. The observation was reproduced when To146-162 treatment was compared with the control peptide. As shown in Figure 4b, mice fed the control peptide were not protected against clinical EAMG, further confirming the specific effect of peptide T $\alpha 146-162$.

AChR-specific T-cell responses from spleen cells are specifically inbibited by oral administration of peptide To146-162. To146-162-treated mice and the remaining 7 animals of the PBS-treated group were sacrificed, and spleen $T$ cells were challenged in vitro with peptide To146-162 and TAChR. Peptide-treated animals had significantly reduced proliferative responses compared with the PBS group (Figure 5a). We repeated the experiment comparing T $\alpha 146-162$-fed animals with control peptide-fed mice $(n=10)$, and a similar reduction of proliferative responses was observed (Figure $5 \mathrm{~b}$ ), confirming the specificity of the tolerogenic effect of peptide treatment.

Oral administration of peptide T $\alpha 146-162$ reduces anti-mouse $A C h R A b$ 's and AChR loss in muscle. We measured the level of anti-mouse AChR Ab's in sera collected from T $\alpha 146-$ 162 -treated mice and control groups. Peptide treatment induced a statistically significant reduction of antimouse AChR Ab's, compared with PBS-fed animals (Figure $6 \mathrm{a} ; P=0.01$ ) and with control peptide-fed mice (Figure $6 \mathrm{~b} ; P=0.03$ ). We also evaluated anti-TAChR Ab's, and a $38.7 \%$ reduction of these Ab's was found in T $\alpha 146-$ 162 -fed mice $(P=0.007)$. This result was confirmed when T $\alpha 146-162$ treatment was repeated and compared with control peptide treatment $(24 \%$ reduction; $P=0.03)$. As a further objective measure of T $\alpha 146-162$ protective effect, we measured the muscle AChR content. As shown in Figure 6c, the muscle AChR content was statistically higher in peptide-fed compared with PBS-fed animals $(P$ $=0.014)$. These results were confirmed when the experiment was repeated and compared with the control pep- 

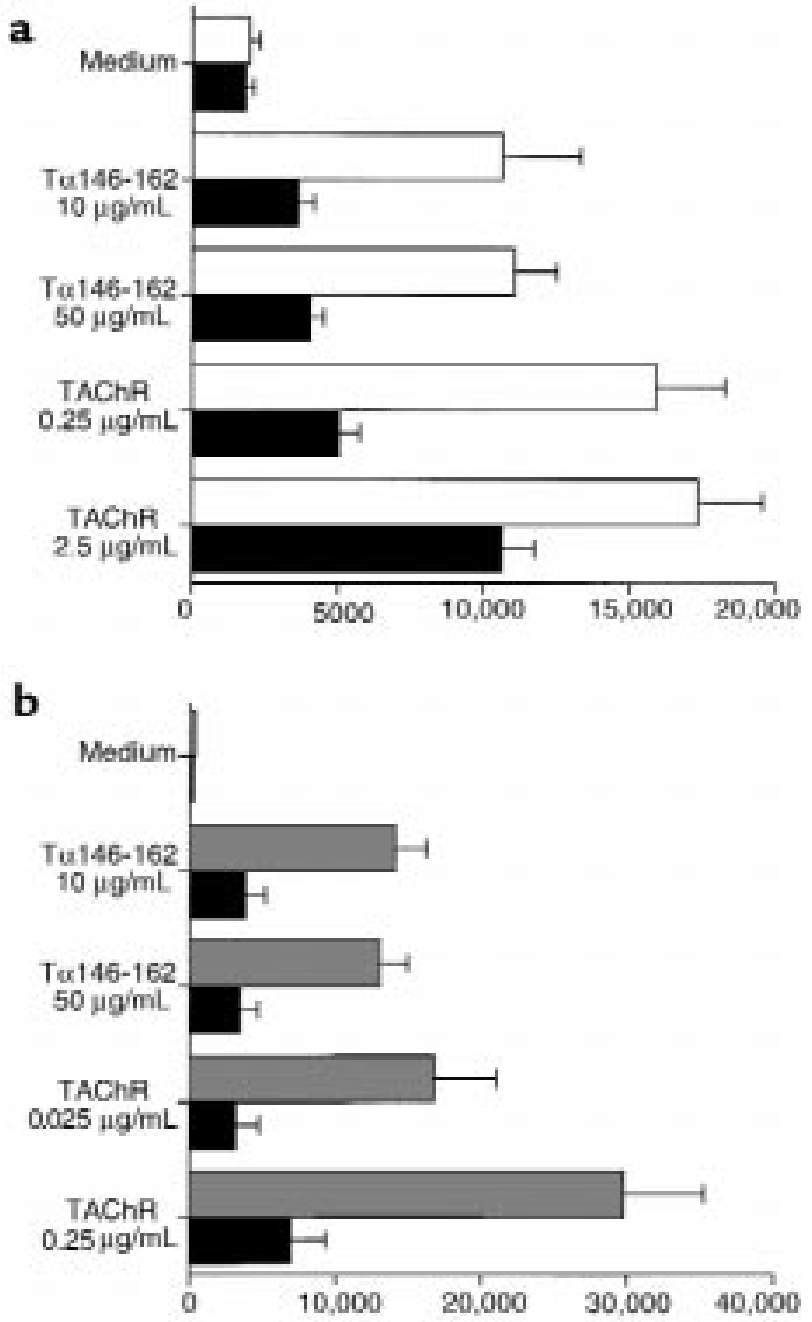

Figure 5

AChR-specific T-cell responses from spleen cells are specifically inhibited by oral administration of peptide To146-162. Proliferative responses of splenic T cells from T $\alpha 146-162-$ fed mice $(n=7)$ were compared with PBS-treated animals $(n=12)(\mathbf{a})$. T cells were chal-

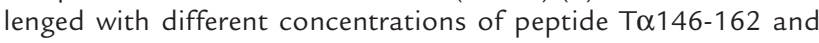
TAChR. Proliferative responses of splenic T cells from peptide-treated animals were significantly reduced at all the concentrations of antigens used. The same pattern was obtained when the experiment was repeated and To146-162-fed animals $(n=12)$ were compared with control peptide-fed mice $(n=10)(\mathbf{b})$. Results are expressed as mean cpm $\pm \mathrm{SEM} ; P<0.01$ for each concentration of antigen used in both experiments.

tide (Figure $6 \mathrm{~d} ; P=0.026$ ). As expected, a slight difference of AChR content between normal mice and T $\alpha 146$ 162-treated animals was found because our treatment did not completely abrogate anti-AChR Ab's; however, such difference was not statistically significant (Figure 6c; $P=0.06$ : Figure $6 \mathrm{~d} ; P=0.1$ ).

Protection against $E A M G$ is associated with downregulation of IgG2 isotype of anti-TAChR Ab's. Anti-TAChR Ab's IgG isotypes were measured in sera from animals treated with peptide T $\alpha 146-162$ or PBS, using secondary Ab's specific for IgG1, IgG2, IgG2a, and IgG2b subclasses. The reduction of anti-TAChR IgG Ab's in peptide-fed mice was because of a reduction of the IgG2 subpopulation, whereas IgG1 were unchanged as shown in Figure 7. This finding is in agreement with the observed pattern of cytokine production.

Modulation of Th1-, Th2-, and Th3-associated cytokines by To146-162 feeding. We measured the production of IFN$\gamma$ and IL-2 as Th1-type cytokines, IL-4 and IL-10 as Th2type cytokines, and TGF- $\beta$ as Th3-type cytokines from TAChR- and T $\alpha 146-162$-stimulated spleen cells from mice treated with Ta146-162 or with the control peptide. As shown in Figure 8, a and b, both IFN- $\gamma$ and IL2 were significantly reduced in supernatants from spleen cells cultured with either TAChR or To146-162, compared with animals treated with the control peptide. IL-10 (Figure 8c) production was reduced significantly only in supernatants from $\mathrm{T}$ cells challenged with TAChR, but not with Ta146-162. IL-4 was not detectable in culture supernatants. Th3 compartment was not influenced by T $\alpha 146-162$ treatment, as indicated by the lack of differences in TGF- $\beta$ levels produced by cultured splenic T cells (Figure $8 \mathrm{~d}$ ).

TGF- $\beta$ mRNA is not increased in PP from T $\alpha 146-$ 162 -treated mice. To further investigate if TGF$\beta$-secreting Th 3 cells were involved in the process of tolerance induction in our model, we isolated PP from the gut of To146-162- and PBS-fed mice 10 days after TAChR immunization. RT-PCR analysis revealed the presence of TGF- $\beta$ mRNA in PBS-fed mice, and its transcription was not different in the peptide-treated group (Figure 9). Densitometric analysis showed a similar ratio of relative expression between the 2 groups (PBS-fed: $0.62 \pm 0.03, n=7$; T $\alpha 146-162$-fed: $0.65 \pm 0.04, n=4$ ).

\section{Discussion}

Oral tolerization has been extensively investigated in cell-mediated models of autoimmune disease with promising results (1). We demonstrate here that B6 mice can be protected from EAMG by the oral administration of a single immunodominant T-cell epitope of the AChR $\alpha$-subunit. Antigen-specific T-cell responses were significantly suppressed by multiple feedings with T $\alpha 146-162$, and the tolerogenic effect was not reproduced in animals fed with a control peptide. To146-162 effect was probably dose-related because the lower dose employed induced tolerance to peptide-specific $\mathrm{T}$ cells only, whereas the higher dose suppressed T lymphocyte proliferation to the native antigen (TAChR) and to a subdominant epitope (To182-198) also, suggesting spreading of tolerance to other T-cell epitopes (16).

Serum levels of autoantibodies cross-reacting with mouse AChR were significantly, even though not completely, reduced by peptide feeding, a specific effect that was not found in mice fed with the control peptide. These findings were paralleled by a lower AChR loss in muscle of treated mice, thus explaining the protective effect of peptide treatment on clinical grounds.

In our model the reduced T- and B-cell autoreactivity to AChR was associated with downregulation of both Th1 and Th2 cytokines. Interestingly, when a lower dose 


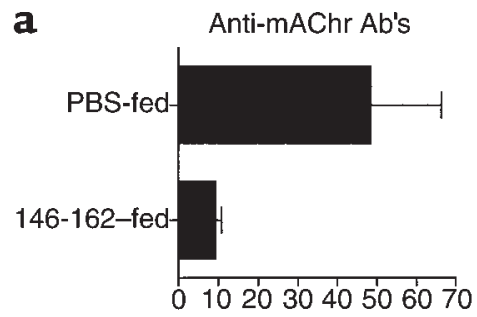

b

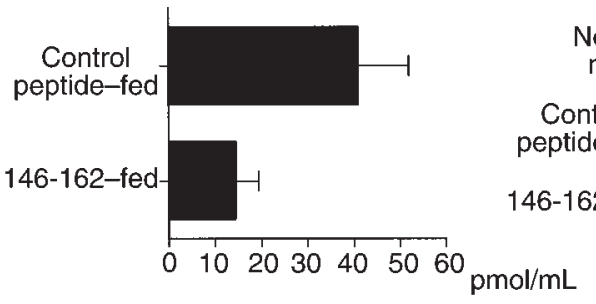

c

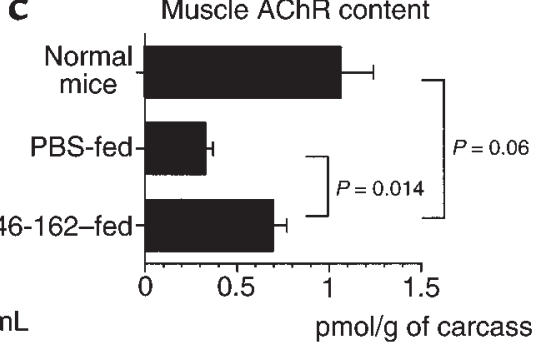

d

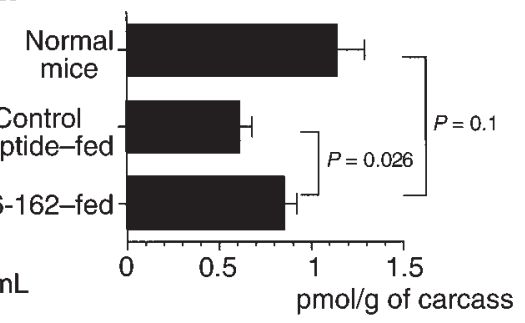

\section{Figure 6}

Oral administration of peptide To146-162 reduces anti-mouse AChR Ab's and muscle AChR loss. Serum levels of anti-AChR Ab's were evaluated in blood samples collected at the time of sacrifice. Anti-mouse AChR Ab levels in peptide-treated mice compared with PBS or control peptide are shown. Peptide treatment induced a statistically significant reduction of anti-mouse AChR Ab's compared with PBS-fed animals $(P=0.01)(\mathbf{a})$ and control peptide-fed animals $(P=0.03)(\mathbf{b})$. Muscle AChR content was higher in peptide-fed compared with PBS-fed animals $(P=0.014)(\mathbf{c})$ but was not statistically different from normal mice $(P=0.06)$. Similar findings were obtained

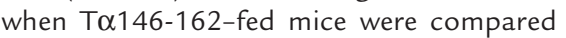
with the control peptide-fed group $(P=0.026)$ (d); the difference between To146-162-fed and normal mice was not statistically significant $(P=0.1)$ of peptide was given, lymph node T-cell responses to TAChR were not suppressed and no significant modifications of cytokine levels were found except for IFN- $\gamma$. On the contrary, when the higher dose was used, the suppression of $\mathrm{T}$ cells to the whole native antigen was associated with a significant reduction of both Th1 and Th2 cytokines. It is worth noting that Wu and coworkers found a similar pattern of cytokine production in B6 mice tolerized subcutaneously with high doses of peptide T $\alpha 146-162$ (16). On the contrary, both anergy and activation of regulatory Th2 cells were involved in B6 mice nasally tolerized by AChR peptides (18).

Previous studies with native antigens or immunodominant fragments provided evidence that clonal anergy and active suppression are the major mechanisms underlying oral tolerance. Antigen dose and schedule of administration play a critical role in the induction of different tolerogenic mechanisms. Indeed, high doses are believed to induce anergy of Th1 and Th2 cells with downregulation of associated cytokines, whereas low doses induce Th2 regulatory cells and increased secretion of TGF- $\beta(1,22)$.

In our model we did not observe increased transcription of TGF- $\beta$ in PP or enhanced secretion by antigenspecific $T$ cells in culture supernatants after peptide treatment. Even though present, TGF- $\beta$-secreting T cells were probably not critical in the process of tolerance induction to AChR, at least with our peptide-feeding protocol. In this regard more detailed studies of the gut-associated lymphoid tissue at different time points from the last feeding are needed to further address this issue. We believe that downregulation of Th1 and Th2 cytokines and AChR-specific T-cell anergy is the main mechanism underlying the induction of AChR tolerance with our feeding protocol. The importance of Th1 downregulation is a key factor in the generation of mucosal tolerance in experimental models of $\mathrm{T}$ cell-mediated autoimmune disease and holds true also for an Ab-mediated disorder such as EAMG. Recent data suggest that both Th1- and Th2-related cytokines are likely to be involved in the pathogenesis of human MG, but, because of different techniques employed, no definite conclusions can be drawn (23). Indeed, it has been reported that production of IFN- $\gamma$ within the neuromuscular junction of transgenic mice induced a myasthenia gravis-like syndrome (24) and that IFN- $\gamma$ knockout mice are resistant to the induction of EAMG (20). These data are in line with the recent observation that nasal tolerance in EAMG can be reversed by nasal administration of IFN- $\gamma(25)$.

The observed changes of anti-AChR Ab isotypes were in keeping with the pattern of cytokine secretion by antigen-specific $\mathrm{T}$ cells because only IgG2 isotypes were reduced by oral tolerization with peptide T $\alpha 146-162$.

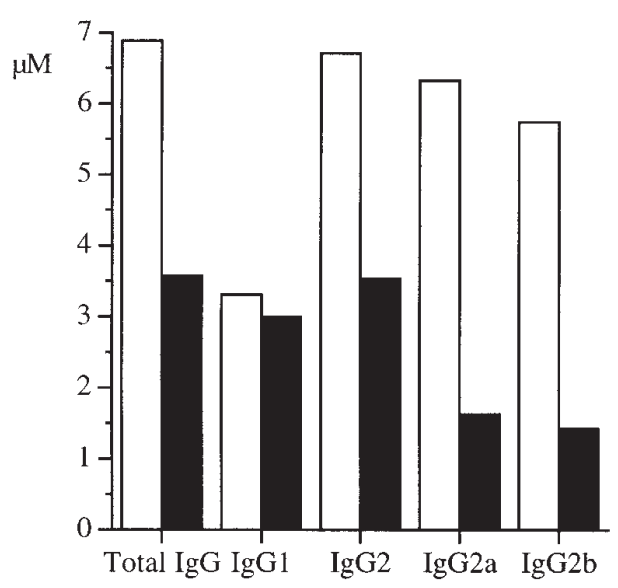

Figure 7

Downregulation of Th1 cytokines was associated with reduction of anti-TAChR IgG2 Ab's. Anti-TAChR antibody lgG isotypes (total lgG, $\lg G 1, \lg G 2$, $\lg G 2 a$, and $\lg G 2 b$ ) were measured at the end of the study in pooled sera collected from animals treated with peptide Ta146-162 (filled columns) or PBS (open columns). The observed reduction in anti-TAChR IgG Ab's in peptide-fed mice was due to reduction of the $\lg G 2$ subpopulation, whereas $\lg G 1$ were unchanged. 

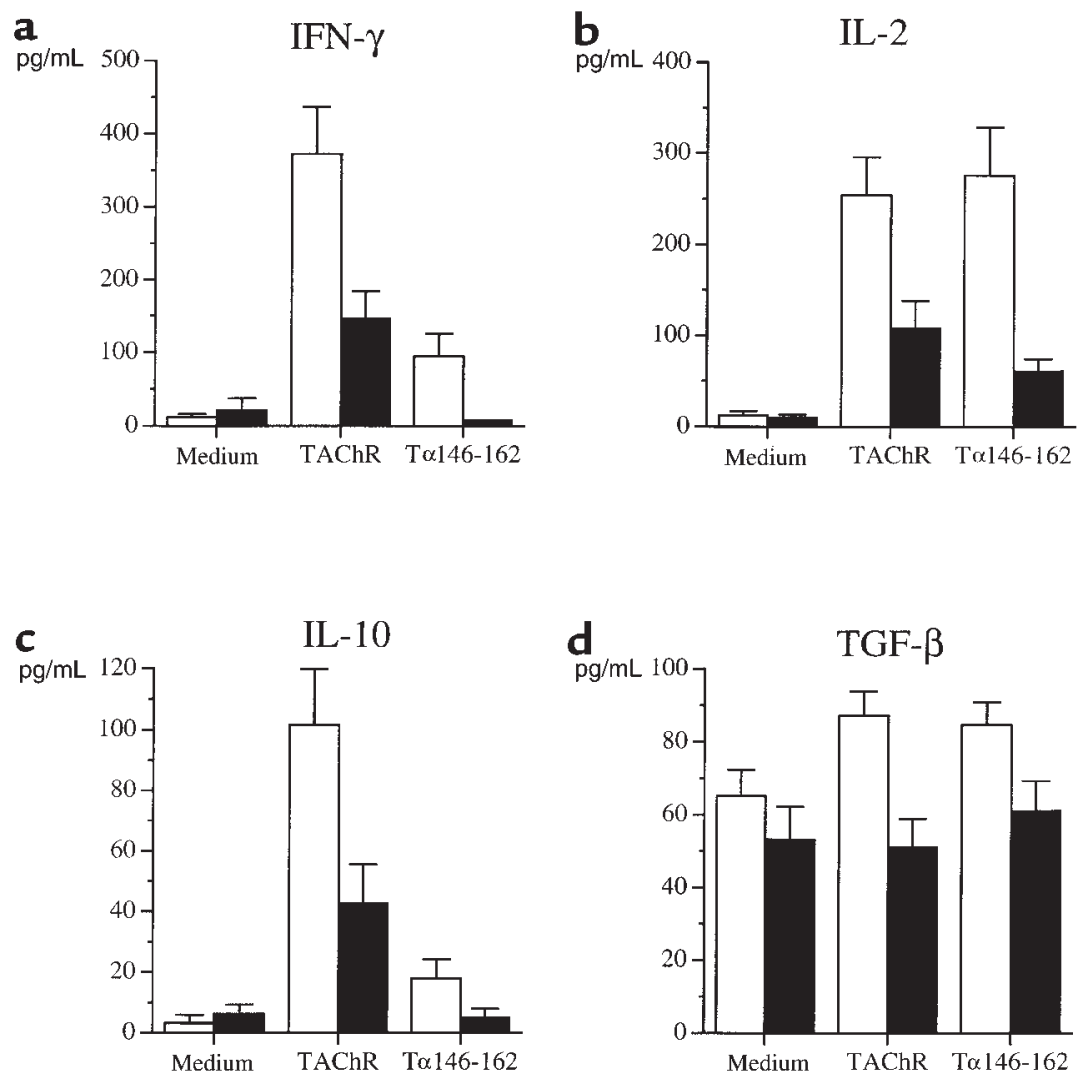

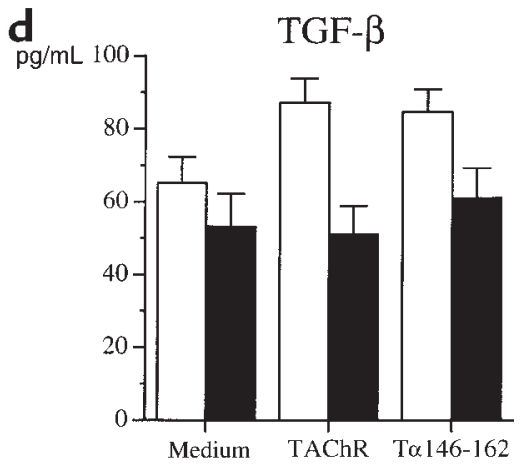

\section{Figure 8}

Protection against clinical EAMG by T $\alpha 146-$ 162 feeding is associated with downregulation ofT-helper cytokines. Peptide treatment significantly reduced IFN- $\gamma$ and IL-2 (Th1type cytokines) production from spleen $\mathrm{T}$ cells cultured with TAChR (IFN- $\gamma, P=0.007$; IL-2, $P=0.0084)$ or T $\alpha 146-162$ (IFN- $\gamma, P=$ 0.01 ; IL-2, $P=0.0004$ ) compared with animals treated with the control peptide ( $\mathbf{a}$ and b). IL-10 production was significantly reduced only in supernatants from $T$ cells challenged with TAChR $(P=0.015)$ but not with To146-162 $(P=0.07)($ c). No production of TGF- $\beta$ was observed in T-cell super-

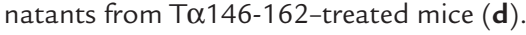

The pathogenetic role of IgG $2 a$ and $2 b$ anti-AChR Ab isotypes has been reported recently in studies performed in B6 mice compared with the EAMG-resistant strain bm12 (26), as well as in IL-12-deficient mice (27).

As reported from low-dose protocol studies in EAE, oral tolerization was mediated by active suppression and increased Th2 cytokines. The issue of inducing an immune deviation from Th1 to Th2 cytokines should be further investigated in T-cell dependent, Ab-mediated diseases. In fact, a word of caution has been raised by the observation of increased titers of autoantibodies to myelin oligodendrocyte glycoprotein (MOG) in marmosets parenterally tolerized to MOG in association with a Th1/Th2 shift; such an increase was, in time, related to exacerbation of the disease (28). Oral administration of large doses of ovalbumin induced autoimmune diabetes in a murine ovalbumin transgenic model (29). When moving from prevention to treatment of EAMG, the possibility that peptide administration might exert a priming rather than protective effect on the immune system should be considered. Indeed, TAChR had a priming effect on B cells when given after EAMG induction in the rat model (10). However, particular attention should be paid to the selection of peptide sequences suitable for tolerization studies. We chose To146-162 because it is a T-cell epitope, is immunodominant in $\mathrm{B} 6$ mice, and it is unlikely to activate AChR-specific B cells because it is not a conformational epitope. Moreover, we did not observe any increase of anti-AChR Ab's after 3 active immunizations in T $\alpha 146-162$-treated mice.
We believe we have shown in this study that the oral administration of a single immunodominant epitope can be used instead of the whole native antigen and that it does not stimulate $\mathrm{B}$ cells. This observation is of primary importance in tolerization studies in Ab-mediated diseases such as EAMG. Considering the high immunogenicity of the AChR, these findings represent a considerable advancement in the field of antigen-specific immunotherapy of this disease. Indeed, a small synthetic fragment eliminates several drawbacks to the use of native AChR, such as availability and immunogenicity for B cells. The results obtained in our model

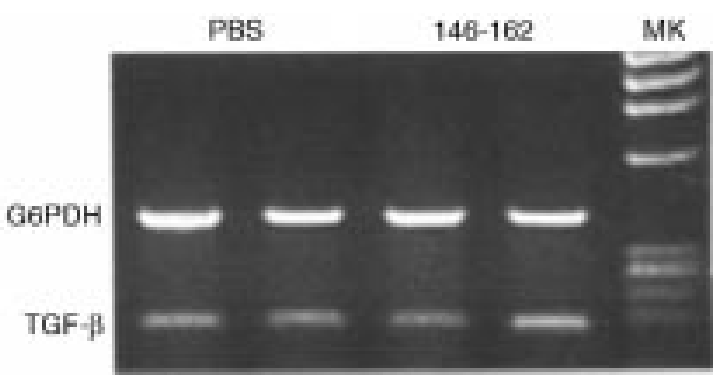

\section{Figure 9}

TGF- $\beta$ transcription is not increased in PP from T $\alpha 146-162-$ fed mice. PP were isolated from the gut of T $\alpha 146-162-\mathrm{fed}(n=4,1 \mathrm{mg} \times 4)$ and PBS-fed mice $(n=7) 10$ days after TAChR immunization. G6PDH mRNA was used as an internal control. Densitometric analysis of TGF- $\beta$ bands did not show significant differences between the 2 treatment groups. (Agarose gel is representative of 2 samples from each group.) 
in which EAMG was prevented by a single immunodominant T-cell epitope of the AChR $\alpha$-subunit suggest its possible application to the treatment of the ongoing disease. We believe that these results will open new perspectives in the antigen-specific immunotherapy of EAMG, the basis for its application to the human disease. Recognition of AChR epitopes by antigen-specific T cells in human MG is heterogeneous and partially conditioned by the technique employed in their detection $(30,31)$. However, recent data indicated that the repertoire of AChR epitopes is probably more restricted than thought previously, suggesting that mucosal tolerization with immunodominant epitopes should be investigated further $(32,33)$.

\section{Acknowledgments}

We are grateful to S. Fuchs (Department of Immunology, Weizmann Institute of Science, Rehovot, Israel) for her suggestions and critical reading of the manuscript. We thank O. Simoncini for help in the anti-AChR Ab's assay and A. Cavallone for technical assistance. This work was partially supported by Telethon Grant 1051 to Carlo Antozzi.

1. Weiner, H.L., et al. 1994. Oral tolerance: immunologic mechanisms and treatment of animal and human organ-specific autoimmune disease by oral administration of autoantigens. Annu. Rev. Immunol. 12:809-837.

2. Wu, H.Y., and Russel, M.W. 1997. Nasal lymphoid tissue, intranasal immunization, and compartmentalization of the common mucosal immune system. Immunol. Res. 16:187-201.

3. Weiner, H.L. 1997. Oral tolerance: immune mechanisms and treatment of autoimmune diseases. Immunol. Today. 18:335-343.

4. Zhu, J., et al. 1998. Prevention of experimental autoimmune neuritis by nasal administration of P2 peptide 57-81. J. Neuropathol. Exp. Neurol. 57:291-301.

5. Marx, A., et al. 1997. Pathogenesis of myasthenia gravis. Virchows Arch. 430:355-364.

6. Bartfeld, D., and Fuchs, S. 1978. Specific immunosuppression of experimental autoimmune myasthenia gravis by denatured acetylcholine receptor. Proc. Natl. Acad. Sci. USA. 75:4006-4010.

7. Wang, Z.Y., Qiao, J., and Link, H. 1993. Suppression of experimental autoimmune myasthenia gravis by oral administration of acetylcholine receptor. J. Neuroimmunol. 44: 209-214.

8. Okumura, S., McIntosh, K., and Drachman, D.B. 1994. Oral administration of acetylcholine receptor: effects on experimental autoimmune myasthenia gravis. Ann. Neurol. 36:704-713.

9. Ma, C.G., et al. 1995. Suppression of experimental autoimmune myasthenia gravis by nasal administration of acetylcholine receptor. J. New roimmunol. 58:51-60.

10. Drachman, D.B., Okumura, S., Adams, R.N., and McIntosh, K. 1996. Oral tolerance in myasthenia gravis. Ann. N.Y. Acad. Sci. 778:258-272.

11. Shi, F.D., Bai, X.F., Li, H.L., Van der Meide, P.H., and Link, H. 1998. Nasal tolerance in experimental autoimmune myasthenia gravis (EAMG): induction of protective tolerance in primed animals. Clin. Exp. Immunol. 111:506-512.

12. Barchan, D., Souroujon, M.C., Im, S.H., Antozzi, C., and Fuch, S. 1999. Antigen-specific modulation of experimental myasthenia gravis: nasal tolerization with recombinant fragment of the human acetylcholine receptor alpha-subunit. Proc. Natl. Acad. Sci. USA. 96:8086-8091.

13. Shenoy, M., Goluzsko, E., and Christadoss, P. 1994. The pathogenic role of acetylcholine receptor $\alpha$ chain epitope within $\alpha 146-162$ in the development of experimental autoimmune myasthenia gravis in C57BL6 mice. Clin. Immunol. Immunopathol. 73:338-343.

14. Bellone, M., Ostlie, N., Lei, S., and Conti-Tronconi, B.M. 1991. Experimental myasthenia gravis in congenic mice: sequence mapping and $\mathrm{H}-2$ restriction of T helper epitopes on the $\alpha$-subunits of Torpedo Californica and murine acetylcholine receptor. Eur. J. Immunol. 21:2303-2310.

15. Christadoss, P., Lindstrom, J., Melvold, R., and Talal, N. 1985. Mutation at I-A beta chain prevents experimental autoimmune myasthenia gravis. Immunogenetics. 21:33-38.

16. Wu, B., Deng, C., Goluszko, E., and Christadoss, P. 1997. Tolerance to a dominant $\mathrm{T}$ cell epitope in the acetylcholine receptor molecule induces epitope spread and suppresses murine myasthenia gravis. J. Immunol. 159:3016-3023.

17. Karachunski, P.I., Ostlie, N.S., Okita, D.K., Garman, R., and Conti-Fine, B.M. 1999. Subcutaneous administration of T-epitope sequences of the acetylcholine receptor prevents experimental myasthenia gravis. J. Neuroimmunol. 93:108-121.

18. Karachunski, P.I., Ostlie, N.S., Okita, D.K., and Conti-Fine, B.M. 1997. Prevention of experimental myasthenia gravis by nasal administration of synthetic acetylcholine receptor T epitope sequences. J. Clin. Invest. 100:3027-3035.

19. Aharonov, A., Tarrab-Hazdai, R., Silman, I., and Fuchs, S. 1977. Immunochemical studies on acetylcholine receptor from Torpedo californica. Immunochemistry. 14:129-137.

20. Balasa, B., et al. 1997. Interferon $\gamma($ IFN- $\gamma$ ) is necessary for the genesis of acetylcholine receptor-induced clinical experimental autoimmune myasthenia gravis in mice. J. Exp. Med. 186:385-391.

21. Lindstrom, J., Einarson, B., and Tzartos, S. 1981. Production and assay of antibodies to acetylcholine receptor. In Methods in enzymology. J. Langone and H. Van Vunakis, editors. Academic Press. New York, NY. 432-460.

22. Strobel, S., and Mowat, A.M. 1998. Immune responses to dietary antigens: oral tolerance. Immunol. Today. 19:173-181.

23. Zhang, G.X., Navikas, V., and Link, H. 1997. Cytokines and the pathogenesis of myasthenia gravis. Muscle Nerve. 20:543-551.

24. Gu, D., et al. 1995. Myasthenia gravis-like syndrome induced by expression of interferon- $\gamma$ in the neuromuscular junction. J. Exp. Med. 181:547-557.

25. Li, H.L., et al. 1998. Nasal tolerance to experimental autoimmune myasthenia gravis: tolerance reversal by nasal administration of minute amounts of interferon-gamma. Clin. Immunol. Immunopathol. 87:15-22.

26. Drachman, D.B., McIntosh, K.R., and Yang, B. 1998. Factors that determine the severity of experimental myasthenia gravis. Ann. NY Acad. Sci. 841:262-282.

27. Moiola, L., et al. 1998. IL-12 is involved in the induction of experimental autoimmune myasthenia gravis, an antibody-mediated disease. Eur. J. Immunol. 28:2487-2497.

28. Genain, C.P., et al. 1996. Late complications of immune deviation therapy in non-human primate. Science. 274:2054-2057.

29. Blanas, E., Carbone, F.R., Allison, J., Miller, J.F.A.P., and Heath, W.R. 1996. Induction of autoimmune diabetes by oral administration of autoantigen. Science. 274:1707-1709.

30. Protti, M.P., Manfredi, A., Horton, R.M., Bellone, M., and Conti-Tronconi, B.M. 1993. Myasthenia gravis: recognition of a human autoantigen at the molecular level. Immunol. Today. 14:363-368.

31. Hawke, S., et al. 1996. Autoimmune T cells in myasthenia gravis: heterogeneity and potential for specific immunotargeting. Immunol. Today. 17:307-311.

32. Wang, Z.Y., Okita, D.K., Howard, J., and Conti-Fine, B.M. 1997. Th1 epitope repertoire on the alpha subunit of human muscle acetylcholine receptor in myasthenia gravis. Neurology. 48:1643-1653.

33. Hill, M., et al. 1999. Early-onset myasthenia gravis: a recurring T cell epitope in the adult-specific acetylcholine receptor epsilon subunit presented by the susceptibility allele HLA-DR52a. Ann. Neurol. 45:224-231. 